\title{
Effects of Fast Ions and an External Inductive Electric Field on the Neoclassical Parallel Flow, Current and Rotation in General Toroidal Systems
}

\author{
Noriyoshi Nakajima and Masao Okamoto \\ National Institute for Fusion Science, Nagoya 464-01.
}

(Received April 15, 1992/Revised Manuscript Received May 23, 1992)

\begin{abstract}
Effects of external momentum sources, i.e., fast ions produced by the neutral beam injection and an external inductive electric field, on the neoclassical ion parallel flow, current, and rotation are analytically investigated for a simple plasma in general toroidal systems. It is shown that the contribution of the external sources to the ion parallel flow becomes large as the collision frequency of thermal ions increases because of the momentum conservation of Coulomb collisions and sharply decreasing viscosity coefficients with collision frequency. As a result, the beam-driven parallel flow of thermal ions becomes comparable to that of electrons in the Pfirsh-Schlüter collisionality regime, whereas in the $1 / \nu$ or banana regime it is smaller than that of electrons by the order of $\sqrt{m_{e} / m_{i}}\left(m_{e}\right.$ and $m_{i}$ are electron and ion masses). This beam-driven ion parallel flow can not produce a large beam-driven current because of the cancellation with electron parallel flow, but produces a large toroidal rotation of ions. As both electron and ions approach the PfirshSchlüter collisionality regime the contribution of thermodynamical forces becomes negligibly small and the large toroidal rotation of ions is predominated by the beam-driven component in the non-axisymmetric configuration with large helical ripples.
\end{abstract}

Keywords:

neoclassical theory, non-axisymmetric system, fast ions, external inductive field, parallel flow, neoclassical current, plateau collisionality regime, Pfirsh-Schlüter collisionality regime,

\section{Introduction}

In recent experiments of Compact Helical System (CHS) heliotron/torsatron device ${ }^{1)}$ with tangential neutral beam injection, plasma rotations have been measured over a wide range of densities and magnetic field ripples ${ }^{2}$. In CHS central helical ripples along the magnetic axis can vary according to the outward or inward shift of the plasma. If $R_{\text {axis }}=90 \sim 95 \mathrm{~cm}$, where $R_{\mathrm{axis}}$ is the major radius of the magnetic axis in the vacuum field, the central helical ripples are negligibly small and they increase sharply if $R_{\text {axis }}$ exceeds $95 \mathrm{~cm}$. In the vicinity of the region where the helical ripples are vanishingly small the system can be thought to be nearly axisymmetric. It is reported in ref. 2 that the profile of the toroidal rotation is dominated by an anomalous shear viscosity when the field ripples are weak near the axis, while the parallel viscosity is found to be dominant when the ripples are strong enough. The observed parallel viscosities have been concluded to agree with the neoclassical predictions within a factor of 
three. In these experiments both electrons and ions were in the plateau collisionality regime. Plasma rotations or flows are determined by the balance between external momentum sources (or input torque) and damping forces of viscosity and friction. Neoclassical theories including momentum sources have been extensively developed for axisymmetric tokamaks ${ }^{3)}$. Recently the authors have developed the neoclassical theory for the parallel force balance to investigate the flow, current, and rotation of a multispecies plasma with external momentum sources in general toroidal systems including both axisymmetric and non-axisymmetric toroidal devices ${ }^{4}$. As external momentum sources fast ions produced by the tangential neutral beam injection and an external inductive electric field have been considered. In this ref. 4, neoclassical expressions obtained so far in each collisionality regime (ref. 5 in the $1 / \nu$ and Pfirsh-Schlüter regimes and refs. 6 and 7 in the plateau regime) have been unified in terms of the geometric factor. General formula common to all collisionalities have been obtained for neoclassical flow and consequently for parallel current and plasma rotation. This extended neoclassical theory has revealed that in addition to the conventional pressure driven neoclassical current (bootstrap current) the parallel current generated directly by the radial electric field, which dose not exist in the axisymmetric system, exists in the nonaxisymmetric system ${ }^{4}$. Rotations of bulk ions and impurity ions have been investigated for a plasma in the general toroidal system based on ref. 4 and the difference between the two has been clarified ${ }^{8)}$.

In the present paper, we examine the effects of external momentum sources on the neoclassical parallel flow, current, and rotation applying the extended neoclassical theory ${ }^{4)}$ to a simple electron-ion plasma in general toroidal systems. We consider fast ions produced by the tangential neutral beam injection and an inductive electric field as the external momentum sources. Equations for parallel flow, current, and rotation are analytically investigated by using the mass ratio expansion of friction and viscosity coefficients. We concentrate our attention on the high collisionality regime taking into account the operation conditions of the above mentioned CHS experiment. In the neoclassical theory, the parallel particle flows are determined by the parallel momentum and heat flux balance equations, i.e., the balance among the friction, viscosity and external momentum sources. The friction matrix consisting of friction coefficients is not regular because the Coulomb collision conserves the total momenta. The viscosity matrix consisting of viscosity coefficients approaches the singular matrix as the collision frequency becomes large because the viscosity coefficients become very small in the high collisionality regime. As a result of it, the contribution of the external momentum sources to the parallel flow may change drastically according to the collisionality regime.

As is well known, when both ions and electrons are in the $1 / \nu$ or banana collisionality regime, the ion parallel flow driven by the external momentum sources is smaller than that of electrons by $\varepsilon \equiv \sqrt{m_{\mathrm{e}} / m_{\mathrm{i}}}$ when $m_{\mathrm{e}}$ and $m_{\mathrm{i}}$ are masses of electrons and ions, respectively. Of course, the momentum transfer to ions from the external momentum sources is larger than that to electrons by $\varepsilon^{-1}$. Therefore, we see that the light electrons carry the current and the heavy ions carry the momentum in the banana regime. It will be shown, however, that if electrons and ions are in the Pfirsh-Schlüter collisionality regime, the contribution of fast ions becomes so large that the beam-driven parallel flow of ions becomes the same order of that of electrons. Accordingly, the momentum transfer to ions from the fast ions is larger than that to electrons by $\varepsilon^{-2}$. This beam-driven ion parallel flow can not produce a large beam-driven current, because it is canceled out by beam-driven electron parallel flow. As a result of it, the neoclassical parallel current reduces to the usual classical expression, while it can contribute to the rotation of ions significantly. It will be also shown that in the non-axisymmetric toroidal system with large helical ripples the contribution of the thermodynamical forces to the parallel flow is negligibly small and the ion parallel flow and toroidal rotation are predominated by the beam-driven component or the external momentum sources. However, it will be remarked that if the axisymmetry is partially recovered in some region as is the case of CHS 
under an experimental condition, the thermodynamical contribution to the ion flow must be included. A different case where electrons and ions exist in different collisionality regimes will be discussed analytically and the similar results will be obtained.

The organization of this paper is as follows. In $\$ 2$ parallel force balances are given and the singular behavior of friction and viscosity matrices in the high collisionality regime is indicated. The parallel flow, current, and rotation are derived for a simple plasma in the general toroidal system. These are analytically investigated in three special cases, placing emphasis on the high collisionality regime in $\$ 3$. Section 4 is devoted to conclusion and discussion. In Appendix 1 the expressions of flux-surface-averaged parallel frictions and viscosities used in $\S 2$ are given. Appendix 2 gives the definitions of quantities in Eq. (2). The friction and viscosity coefficients are given in Appendix 3.

\section{Parallel Flow, Current, and Rotation Including External Momentum Sources for a Simple Plasma}

Neoclassical theories for the parallel flow, current, and rotation have been extended to a multispecies plasma in general toroidal systems ${ }^{4}$. This extended theory includes the external momentum sources of fast ions produced by the tangential neutral beam injection and an external inductive electric field. In this ref. 4 general expressions, which are common in all collisionality regime ( $1 / \nu$, plateau, and Pfirsh-Schlüter regimes), have been derived for the parallel flow, current, and rotation in terms of the geometric factor. In this section we apply this theory to a simple plasma consisting of electrons and one species ions.

First we concider asymptotic behaviors of viscous and frictional matrices. To determine the flux-surface-averaged parallel flow $\left\langle B u_{/ / a}\right\rangle$ and heat flux $\left\langle B q_{/ / a}\right\rangle$, the flux-surface-averaged parallel momentum and heat flux balance equations are $\mathrm{used}^{3-8)}$ :

$$
\left[\begin{array}{c}
\left\langle\boldsymbol{B} \cdot \boldsymbol{V} \cdot \Pi_{a}\right\rangle \\
-\left\langle\boldsymbol{B} \cdot \nabla \cdot \Theta_{a}\right\rangle
\end{array}\right]=\left[\begin{array}{c}
\left\langle\boldsymbol{B} \cdot \boldsymbol{F}_{a 1}\right\rangle \\
-\left\langle\boldsymbol{B} \cdot \boldsymbol{F}_{a 2}\right\rangle
\end{array}\right]+\left[\begin{array}{c}
\left\langle\boldsymbol{B} \cdot \boldsymbol{F}_{a \mathrm{f} 1}\right\rangle \\
-\left\langle\boldsymbol{B} \cdot \boldsymbol{F}_{a \mathrm{f} 2}\right\rangle
\end{array}\right]+\left[\begin{array}{c}
n_{a} e_{a}\left\langle\boldsymbol{B} \cdot \boldsymbol{E}^{(A)}\right\rangle \\
0
\end{array}\right],
$$

where $\Pi_{a}$ and $\Theta_{a}$ are the stress and heat stress tensors, respectively. $\boldsymbol{F}_{a j}$ and $\boldsymbol{F}_{a \mathrm{f} j}(\boldsymbol{j}=1$ or 2$)$ are frictions of species $a$ with thermal species and fast ions, respectively. $\boldsymbol{E}^{(A)}$ indicates the external inductive electric field. The expressions of the flux-surface-averaged parallel quantities can be found in ref. 4 and repeated in Appendix 1.

Substitution of Eqs. (A. 1) and (A. 4)-(A. 5) in Appendix 1 into Eq. (1) gives the following linear algebraic equation for $X$ :

$$
\boldsymbol{\mu}(\boldsymbol{X}+\boldsymbol{G})=\boldsymbol{L}_{\mathrm{t}} \boldsymbol{X}+\boldsymbol{F}_{\mathrm{f}}+\boldsymbol{E}_{\mathrm{A}},
$$

where $\boldsymbol{X}$ is the parallel flow and parallel heat flux to be solved, $\boldsymbol{\mu}$ and $\boldsymbol{L}_{\mathrm{t}}$ indicate the viscous and frictional matrices, which are constructed in terms of viscosity and friction coefficients, respectively. $\boldsymbol{G}, \boldsymbol{F}_{\mathrm{f}}$ and $\boldsymbol{E}_{\mathrm{A}}$ express the thermodynamical force, the friction with fast ions, and the external inductive electric field, respectively. The definition of each quantity is given in Appendix 2. The solutions to Eq. (2) are given by

$$
\boldsymbol{X}=\left(\boldsymbol{L}_{\mathrm{t}}-\boldsymbol{\mu}\right)^{-1} \boldsymbol{\mu} G-\left(\boldsymbol{L}_{\mathrm{t}}-\boldsymbol{\mu}\right)^{-1}\left(\boldsymbol{F}_{\mathrm{f}}+\boldsymbol{E}_{\mathrm{A}}\right) .
$$

We can see from Ep. (3) that the parallel flow and heat flux consist of two terms: one is due to the thermodynamical force and the other is due to the external momentum sources. The friction force conserves the total momenta as shown by Eq. (A. 3) in Appendix 1, which is expressed in terms of $\boldsymbol{L}_{\mathrm{t}}$ as follows:

$$
\left|\boldsymbol{L}_{\mathrm{t}}\right|=0 \text {. }
$$

As is mentioned in Appendix 1, the viscosity coefficients decrease so rapidly as the collision frequency increases that they become small in the plateau regime and very small in the PfirshSchlüter regime. From this property and the total momentum conservation given by Eq. (4), we see, in the limit of high collisionality regime (plateau or Pfirsh-Schlüter regime), 


$$
\boldsymbol{\mu} \rightarrow 0, \quad\left(\boldsymbol{L}_{\mathrm{t}}-\boldsymbol{\mu}\right)^{-1} \rightarrow \infty, \quad\left(\boldsymbol{L}_{\mathrm{t}}-\boldsymbol{\mu}\right)^{-1} \boldsymbol{\mu} \rightarrow \text { finite } .
$$

Therefore, in these high collisionality regimes, the effects of external momentum sources (the second term on the right-hand side of Eq. (3)) may become significant. The parallel viscosity and heat visosity given by Eq. (A. 5) are calculated by using the gyro-averaged distribution function. There is another type of viscosity, i.e., gyroviscosity, which is obtained by using the gyrovarying distribution function. The gyroviscosity dose not depend on the collisionality regime. The order of the gyroviscosity of particle spcies $a$ is smaller than the parallel viscosity by the order of $O\left(\rho_{a} / L\right)$ for $\rho_{a} / L \ll 1$, where $\rho_{a}$, and $L$ are the Larmor radius of particle species $a$, and the characteristic length of the system, respectively, hence the significancy of the effects of the external momentum sources may not change in the high collisionality regime even if the gyroviscosity is included.

To clarify the effects of the external sources on the parallel flow, current, and rotation, let us consider a simple electron-ion plasma. Assuming $T_{\mathrm{e}} \sim T_{\mathrm{i}}$ and using

$$
\varepsilon \equiv \frac{\frac{n_{\mathrm{e}} m_{\mathrm{e}}}{\tau_{\mathrm{ee}}}}{\frac{n_{\mathrm{i}} m_{\mathrm{i}}}{\tau_{\mathrm{ii}}}} \simeq \sqrt{\frac{m_{\mathrm{e}}}{m_{\mathrm{i}}}} \frac{1}{Z_{\text {eff }}^{2}} \ll 1,
$$

as the expansion parameter, we calculate the elements of matrices $\boldsymbol{L}_{\mathrm{t}}$ and $\boldsymbol{\mu}$ up to the order of $\varepsilon$. Substitution of this result into Eq. (3) gives the parallel flows for electrons and ions, the parallel current, and the poloidal and toroidal rotations for the simple plasma. We use the Boozer coordinates $(\phi, \theta, \zeta)$, where $\phi$ is defined as the toroidal flux divided by $2 \pi, \theta$ and $\zeta$ are, respectively, the poloidal and toroidal angles. For the simple plasma the parallel flows for electrons and ions are given by, respectively,

$$
\begin{aligned}
\left\langle B u_{/ / \mathrm{e}}\right\rangle & =\left\langle G_{\mathrm{BS}}\right\rangle_{\mathrm{e}}\left\{\left(L_{11}^{(0)}+L_{11}^{(1)}\right)\left(-\frac{P_{\mathrm{e}}^{\prime}}{e n_{\mathrm{e}}}+\phi^{\prime}\right)+\left(L_{12}^{(0)}+L_{12}^{(1)}\right) \frac{T_{\mathrm{e}}^{\prime}}{e}\right\} \\
& -\left(1+L_{11}^{(0)}+L_{11}^{(1)}\right)\left\langle G_{\mathrm{BS}}\right\rangle_{\mathrm{i}}\left\{\frac{P_{\mathrm{i}}^{\prime}}{e n_{\mathrm{e}}}+\phi^{\prime}+L_{34}^{(1)} \frac{T_{\mathrm{i}}^{\prime}}{e Z_{\mathrm{eff}}}\right\} \\
& +\left(1+L_{11}^{(0)}+L_{11}^{(1)}\right)\left\{\frac{Z_{\mathrm{f}}}{Z_{\mathrm{eff}}}-Z_{\mathrm{f}}\left[1+\left(1+\frac{m_{\mathrm{i}}}{m_{\mathrm{f}}}\right)\left(\frac{v_{\mathrm{c}}}{\bar{u}_{\mathrm{f}}}\right)^{3}\right] N_{33}^{(1)}\right\} \frac{Z_{\mathrm{f}} n_{\mathrm{f}}}{n_{\mathrm{e}}}\left\langle B u_{/ / \mathrm{f}}\right\rangle \\
& -\left(1+L_{11}^{(1)}\right)\left\{\Lambda_{\mathrm{NC}}^{(0)}+\Lambda_{\mathrm{NC}}^{(1)}\right\} \frac{e \tau_{\mathrm{ei}}}{m_{\mathrm{e}}}\left\langle B E_{/ /}^{(A)}\right\rangle, \\
\left\langle B u_{/ / \mathrm{i}}\right\rangle & =\left\langle G_{\mathrm{BS}}\right\rangle_{\mathrm{e}}\left\{L_{11}^{(1)}\left(-\frac{P_{\mathrm{e}}^{\prime}}{e n_{\mathrm{e}}}+\phi^{\prime}\right)+L_{32}^{(1)} \frac{T_{\mathrm{e}}^{\prime}}{e}\right\} \\
& -\left(1+L_{11}^{(1)}\right)\left\langle G_{\mathrm{BS}}\right\rangle_{\mathrm{i}}\left\{\frac{P_{\mathrm{i}}^{\prime}}{e n_{\mathrm{e}}}+\phi^{\prime}+L_{34}^{(0)} \frac{T_{\mathrm{i}}^{\prime}}{e Z_{\mathrm{eff}}}\right\} \\
& +\left\{L_{11}^{(1)} \frac{Z_{\mathrm{f}}}{Z_{\mathrm{eff}}}-\left(1+L_{11}^{(1)}\right) Z_{\mathrm{f}}\left[1+\left(1+\frac{m_{\mathrm{i}}}{m_{\mathrm{f}}}\right)\left(\frac{v_{\mathrm{c}}}{\bar{u}_{\mathrm{f}}}\right)^{3}\right] N_{33}^{(1)}\right\} \frac{Z_{\mathrm{f}} n_{\mathrm{f}}}{n_{\mathrm{e}}}\left\langle B u_{/ / \mathrm{f}}\right\rangle \\
& +Z_{\mathrm{eff}} L_{11}^{(0)} N_{33}^{(1)} \frac{e \tau_{\mathrm{ei}}}{m_{\mathrm{e}}}\left\langle B E_{/ /}^{(A)}\right\rangle .
\end{aligned}
$$

The parallel current is given by

$$
\begin{aligned}
\left\langle B J_{/ /}\right\rangle & =e n_{\mathrm{e}}\left\{\left\langle B u_{/ / \mathrm{i}}\right\rangle-\left\langle B u_{/ / \mathrm{e}}\right\rangle\right\}+\left\langle B J_{/ / \mathrm{f}}\right\rangle \\
& =L_{11}^{(0)}\left\{\left(\left\langle G_{\mathrm{BS}}\right\rangle_{\mathrm{i}}-\left\langle G_{\mathrm{BS}}\right\rangle_{\mathrm{e}}\right) e n_{\mathrm{e}} \phi^{\prime}+\left\langle G_{\mathrm{BS}}\right\rangle_{\mathrm{e}} P_{\mathrm{e}}^{\prime}+\left\langle G_{\mathrm{BS}}\right\rangle_{\mathrm{i}} P_{\mathrm{i}}^{\prime}+\left\langle G_{\mathrm{BS}}\right\rangle_{\mathrm{i}} L_{34}^{(0)} n_{\mathrm{i}} T_{\mathrm{i}}^{\prime}\right\} \\
& -\left(L_{12}^{(0)}+L_{12}^{(1)}-L_{32}^{(1)}\right)\left\langle G_{\mathrm{BS}}\right\rangle_{\mathrm{e}} n_{\mathrm{e}} T_{\mathrm{e}}^{\prime} \\
& +\left\{1-\left(1+L_{11}^{(0)}\right) \frac{Z_{\mathrm{f}}}{Z_{\mathrm{eff}}}+L_{11}^{(0)} Z_{\mathrm{f}}\left[1+\left(1+\frac{m_{\mathrm{i}}}{m_{\mathrm{f}}}\right)\left(\frac{v_{\mathrm{c}}}{\bar{u}_{\mathrm{f}}}\right)^{3}\right] N_{33}^{(1)}\right\}\left\langle B J_{/ / \mathrm{f}}\right\rangle \\
& +\left\{\left(1+L_{11}^{(1)}\right)\left(\Lambda_{\mathrm{NC}}^{(0)}+\Lambda_{\mathrm{NC}}^{(1)}\right)+Z_{\mathrm{eff}} L_{11}^{(0)} N_{33}^{(1)}\right\} \frac{e^{2} n_{\mathrm{e}} \tau_{\mathrm{ei}}}{m_{\mathrm{e}}}\left\langle B E_{/ /}^{(A)}\right\rangle .
\end{aligned}
$$

The toroidal and poloidal rotations are given by, respectively, 


$$
\begin{aligned}
& \left\langle\vec{u}_{\mathrm{a}} \cdot \nabla \zeta\right\rangle=\frac{1}{J+t I}\left\langle B u_{/ / \mathrm{a}}\right\rangle-\frac{I}{J+t I}\left(\frac{1}{e_{\mathrm{a}} n_{\mathrm{a}}} \frac{d P_{\mathrm{a}}}{d \phi}+\frac{d \phi}{d \phi}\right), \\
& \left\langle\vec{u}_{\mathrm{a}} \cdot \nabla \theta\right\rangle=\frac{\iota}{J+t I}\left\langle B u_{/ / \mathrm{a}}\right\rangle+\frac{J}{J+t I}\left(\frac{1}{e_{\mathrm{a}} n_{\mathrm{a}}} \frac{d P_{\mathrm{a}}}{d \phi}+\frac{d \phi}{d \phi}\right) .
\end{aligned}
$$

In Eqs. (7) to (11) transport coefficients are given by

$$
\begin{aligned}
L_{11}^{(0)} & =\left\{\bar{\mu}_{\mathrm{e} 1}\left(\bar{l}_{22}^{\mathrm{ee}}-\bar{\mu}_{\mathrm{e} 3}\right)-\bar{\mu}_{\mathrm{e} 2}\left(\bar{l}_{12}^{\mathrm{ee}}-\bar{\mu}_{\mathrm{e} 2}\right)\right\} \frac{1}{D}, \\
L_{11}^{(1)} & =\frac{D_{\mathrm{e} 2}}{D} N_{33}^{(1)}, \\
L_{12}^{(0)} & =\left\{\bar{\mu}_{\mathrm{e} 2} \bar{l}_{22}^{\mathrm{ee}}-\bar{\mu}_{\mathrm{e} 3} \bar{l}_{12}^{\mathrm{ee}}\right\} \frac{1}{D}, \\
L_{12}^{(1)} & =\left\{\bar{l}_{11}^{\mathrm{ee}} \bar{l}_{22}^{\mathrm{ee}}-\left(\bar{l}_{12}^{\mathrm{ee}}\right)^{2}\right\} \bar{\mu}_{\mathrm{e} 2} \frac{1}{D} N_{33}^{(1)}, \\
L_{34}^{(0)} & =\bar{l}_{22}^{\mathrm{ii}} \bar{\mu}_{\mathrm{i} 2} \frac{1}{D_{\mathrm{i} 1}}, \\
L_{32}^{(1)} & =\left\{\bar{\mu}_{\mathrm{e} 2}\left[\bar{l}_{11}^{\mathrm{ee}} \bar{l}_{22}^{\mathrm{ee}}-\left(\bar{l}_{12}^{\mathrm{ee}}\right)^{2}\right]-\bar{l}_{12}^{\mathrm{ee}}\left[\bar{\mu}_{\mathrm{e} 1} \bar{\mu}_{\mathrm{e} 3}-\left(\bar{\mu}_{\mathrm{e} 2}\right)^{2}\right]\right\} \frac{1}{D} N_{33}^{(1)}, \\
N_{33}^{(1)} & =\frac{D_{\mathrm{i} 2}}{D_{\mathrm{i} 1}} \varepsilon \\
\Lambda_{\mathrm{NC}}^{(0)} & =\bar{l}_{11}^{\mathrm{ee}}\left\{\bar{l}_{22}^{\mathrm{ee}}-\bar{\mu}_{\mathrm{e} 3}\right\} \frac{1}{D_{\mathrm{e} 1}}, \\
\Lambda_{\mathrm{NC}}^{(1)} & =-\bar{l}_{11}^{\mathrm{ee}} \bar{l}_{12}^{\mathrm{ee}} \bar{\mu}_{\mathrm{e} 2} \frac{1}{D_{\mathrm{e} 1}} N_{33}^{(1)}, \\
D & =D_{\mathrm{e} 1}-D_{\mathrm{e} 2} N_{33}^{(1)}, \\
D_{\mathrm{e} 1} & =\left(\bar{l}_{11}^{\mathrm{ee}}-\bar{\mu}_{\mathrm{e} 1}\right)\left(\bar{l}_{22}^{\mathrm{ee}}-\bar{\mu}_{\mathrm{e} 3}\right)-\left(\bar{l}_{12}^{\mathrm{ee}}-\bar{\mu}_{\mathrm{e} 2}\right)^{2}, \\
D_{\mathrm{e} 2} & =\left[\bar{l}_{11}^{\mathrm{ee}} \bar{l}_{22}^{\mathrm{ee}}-\left(\bar{l}_{12}^{\mathrm{ee}}\right)^{2}\right] \bar{\mu}_{\mathrm{e} 1}-\bar{l}_{11}^{\mathrm{ee}}\left[\bar{\mu}_{\mathrm{e} 1} \bar{\mu}_{\mathrm{e} 3}-\left(\bar{\mu}_{\mathrm{e} 2}\right)^{2}\right], \\
D_{\mathrm{i} 1} & =-\bar{\mu}_{\mathrm{i} 1}\left(\bar{l}_{22}^{\mathrm{ii}}-\bar{\mu}_{\mathrm{i} 3}\right)-\left(\bar{\mu}_{\mathrm{i} 2}\right)^{2}, \\
D_{\mathrm{i} 2} & =\bar{l}_{22}^{\mathrm{i}}-\bar{\mu}_{\mathrm{i} 3},
\end{aligned}
$$

and $t, 2 \pi J$ and $2 \pi I$ are the rotational transform, the total poloidal current outside a flux surface, and the total toroidal current inside a flux surface, respectively. $\left\langle G_{\mathrm{BS}}\right\rangle_{\mathrm{e}}$ and $\left\langle G_{\mathrm{BS}}\right\rangle_{\mathrm{i}}$ are geometric factors for electrons and ions ${ }^{4,5)}$ reflecting the breaking of axisymmetry and their expressions can be found in ref. 4 . The normalized friction coefficients $\bar{l}_{i j}^{\mathrm{ee}}(i, j=1,2)$ and $\bar{l}_{22}^{i i}$ are given in Appendix 3, which do not depend on the collisionality. $\bar{\mu}_{\mathrm{e} k}$ and $\bar{\mu}_{\mathrm{i} k}(k=1,2,3)$ are the normalized viscosity coefficients of electrons and ions, respectively, the asymptotic forms of which in the $1 / \nu$ or banana, plateau, and Pfirsh-Schlüter regime are also given in Appendix 3 . In the $1 / \nu$ collisionality regime, the normalized viscosity coefficients are proportional to $f_{\mathrm{t}} / f_{\mathrm{c}}$ where $f_{\mathrm{t}}$ is the fraction of trapped particles and $f_{\mathrm{c}}=1-f_{\mathrm{t}}$. In the plateau regime, they are proportional to $\lambda_{a} / \lambda_{\mathrm{PL}}$ where $\lambda_{a}$ and $\lambda_{\mathrm{PL}}$ are the mean free path of species $a$ and the characteristic length of the magnetic field in the plateau regime, respectively. In the Pfirsh-Schlüter regime, the normalized viscosity coefficients are proportional to $\left(\lambda_{a} / \lambda_{\mathrm{PS}}\right)^{2}$, where $\lambda_{\text {PS }}$ is the characteristic length of the magnetic field in the Pfirsh-Schlüter regime. As the mean free path is inversely proportional to the collision frequency $\left(\lambda_{a} \propto 1 / \nu_{a a}\right)$, the normalized viscosity coefficients appearing in Eq. (12) decrease sharply with increasing collision frequency. Note that teams with superscript (1) are proportional to $\varepsilon$.

\section{Special Limiting Cases}

To understand effects of the collisionality on the parallel flows due to the external momentum sources, we consider analytically three asymptotic cases, i.e., 1) the case where both electrons and ions exist in the $1 / \nu$ or banana collisionality regime, which is realized by $\bar{\mu}_{\mathrm{e} k}, \bar{\mu}_{\mathrm{i} k}$ $\gg \varepsilon$, 2) the case where both electrons and ions exist in the Pfirsh-Schlüter regime, which is 
realized by $\bar{\mu}_{e k}, \bar{\mu}_{i k} \sim \varepsilon$, and 3) the case where electrons and ions exist in the $1 / \nu$ and PfirshSchlüter regime, respectively, which is realized by $\bar{\mu}_{e k} \gg \bar{\mu}_{i k} \sim \varepsilon$. Note that for the plateau collisionality regime we need different ordering with respect to $\bar{\mu}_{e k}$ and $\bar{\mu}_{i k}$. In this section, however, we exclude such a case.

At first, we consider the situation where both electrons and ions exist in the $1 / \nu$ or banana collisionality regime $\left(\bar{\mu}_{\mathrm{e} k}, \bar{\mu}_{\mathrm{i} k} \gg \varepsilon\right)$. As is seen from Eqs. (7) and (8) thermodynamical forces and external momentum sources (fast ions and the external inductive field) drive the flows of electrons and ions. By retaining the leading terms for each driving term, following equations are obtained:

$$
\begin{gathered}
\left\langle B u_{/ / \mathrm{e}}\right\rangle=\left\langle G_{\mathrm{BS}}\right\rangle_{\mathrm{e}}\left\{L_{11}^{(0)}\left(-\frac{P_{\mathrm{e}}^{\prime}}{e n_{\mathrm{e}}}+\phi^{\prime}\right)+L_{12}^{(0)} \frac{T_{\mathrm{e}}^{\prime}}{e}\right\} \\
-\left(1+L_{11}^{(0)}\right)\left\langle G_{\mathrm{BS}}\right\rangle_{\mathrm{i}}\left\{\frac{P_{\mathrm{i}}^{\prime}}{e n_{\mathrm{e}}}+\phi^{\prime}+L_{34}^{(0)} \frac{T_{\mathrm{i}}^{\prime}}{e Z_{\mathrm{eff}}}\right\} \\
+\left(1+L_{11}^{(0)}\right) \frac{Z_{\mathrm{f}}}{Z_{\mathrm{eff}}} \frac{Z_{\mathrm{f}} n_{\mathrm{f}}}{n_{\mathrm{e}}}\left\langle B u_{/ / \mathrm{f}}\right\rangle \\
-\Lambda_{\mathrm{NC}}^{(0)} \frac{e \tau_{\mathrm{ei}}}{m_{\mathrm{e}}}\left\langle B E_{/ /}^{(A)}\right\rangle, \\
\left\langle B u_{/ / \mathrm{i}}\right\rangle=-\left\langle G_{\mathrm{BS}}\right\rangle_{\mathrm{i}}\left\{\frac{P_{\mathrm{i}}^{\prime}}{e n_{\mathrm{e}}}+\phi^{\prime}+L_{34}^{(0)} \frac{T_{\mathrm{i}}^{\prime}}{e Z_{\mathrm{eff}}}\right\} \\
+N_{33}^{(1)}\left\{\frac{D_{\mathrm{e} 2}}{D_{\mathrm{e} 1}} \frac{Z_{\mathrm{f}}}{Z_{\mathrm{eff}}}-Z_{\mathrm{f}}\left[1+\left(1+\frac{m_{\mathrm{i}}}{m_{\mathrm{f}}}\right)\left(\frac{v_{\mathrm{c}}}{\bar{u}_{\mathrm{f}}}\right)^{3}\right]\right\} \frac{Z_{\mathrm{f}} n_{\mathrm{f}}}{n_{\mathrm{e}}}\left\langle B u_{/ / \mathrm{f}}\right\rangle \\
+Z_{\mathrm{eff}} L_{11}^{(0)} N_{33}^{(1)} \frac{e \tau_{\mathrm{ei}}}{m_{\mathrm{e}}}\left\langle B E_{/ /}^{(A)}\right\rangle, \\
\left\langle B J_{/ /}\right\rangle=L_{11}^{(0)}\left\{\left(\left\langle G_{\mathrm{BS}}\right\rangle_{\mathrm{i}}-\left\langle G_{\mathrm{BS}}\right\rangle_{\mathrm{e}}\right) e n_{\mathrm{e}} \phi^{\prime}+\left\langle G_{\mathrm{BS}}\right\rangle_{\mathrm{e}} P_{\mathrm{e}}^{\prime}+\left\langle G_{\mathrm{BS}}\right\rangle_{\mathrm{i}} P_{\mathrm{i}}^{\prime}+\left\langle G_{\mathrm{BS}}\right\rangle_{\mathrm{i}} L_{34}^{(0)} n_{\mathrm{i}} T_{\mathrm{i}}^{\prime}\right\} \\
-L_{12}^{(0)}\left\langle G_{\mathrm{BS}}\right\rangle_{\mathrm{e}} n_{\mathrm{e}} T_{\mathrm{e}}^{\prime} \\
+\left\{1-\left(1+L_{11}^{(0)}\right) \frac{Z_{\mathrm{f}}}{Z_{\mathrm{eff}}}\right\}\left\langle B J_{/ / \mathrm{f}}\right\rangle \\
+\Lambda_{\mathrm{NC}}^{(0)} \frac{e^{2} n_{\mathrm{e}} \tau_{\mathrm{ei}}}{m_{\mathrm{e}}}\left\langle B E_{/ /}^{(A)}\right\rangle,
\end{gathered}
$$

where

$$
\begin{aligned}
L_{11}^{(0)} & =\left\{\bar{\mu}_{\mathrm{e} 1}\left(\bar{l}_{22}^{\mathrm{ee}}-\bar{\mu}_{\mathrm{e} 3}\right)-\bar{\mu}_{\mathrm{e} 2}\left(\bar{l}_{12}^{\mathrm{ee}}-\bar{\mu}_{\mathrm{e} 2}\right)\right\} \frac{1}{D_{\mathrm{e} 1}}, \\
L_{12}^{(0)} & =\left\{\bar{\mu}_{\mathrm{e} 2} \bar{l}_{22}^{\mathrm{ee}}-\bar{\mu}_{\mathrm{e} 3} \bar{l}_{12}^{\mathrm{ee}}\right\} \frac{1}{D_{\mathrm{e} 1}}, \\
L_{34}^{(0)} & =\bar{l}_{22}^{\mathrm{ii}} \bar{\mu}_{\mathrm{i} 2} \frac{1}{D_{\mathrm{i} 1}}, \\
N_{33}^{(1)} & =\frac{D_{\mathrm{i} 2}}{D_{\mathrm{i} 1}} \varepsilon, \\
\Lambda_{\mathrm{NC}}^{(0)} & =\bar{l}_{11}^{\mathrm{ee}}\left\{\bar{l}_{22}^{\mathrm{ee}}-\bar{\mu}_{\mathrm{e} 3}\right\} \frac{1}{D_{\mathrm{e} 1}} .
\end{aligned}
$$

$D_{\mathrm{e} 1}, D_{\mathrm{e} 2}, D_{\mathrm{i} 1}$, and $D_{\mathrm{i} 2}$ are the same as those in Eq. (12). In Eq. (16), $N_{33}^{(1)}$ is smaller than others by the order of $\varepsilon$. Comparing Eq. (13) with Eq. (14), we see that the parallel flow of electrons due to fast ions and the external inductive field is larger than that of ions by the order of $\varepsilon$. As shown in Eq. (15), the beam-driven current and Spitzer current are predominated by light electrons. However, it should be noted that the parallel momentum given to electrons, $m_{\mathrm{e}}\left\langle B u_{/ / \mathrm{e}}\right\rangle$, from both fast ions and the external inductive field is smaller than that of ions, $m_{\mathrm{i}}\left\langle B u_{/ / \mathrm{i}}\right\rangle$, by the order of $\varepsilon$. Thus, we have usual results.

Secondly, let us consider the case where both electrons and ions exist in the PfirshSchlüter regime, i.e., $\bar{\mu}_{\mathrm{e} k} \sim \bar{\mu}_{\mathrm{i} k} \sim \varepsilon$. In this case, Eqs. (7)-(9) become as follows: 


$$
\begin{aligned}
\left\langle B u_{/ / \mathrm{e}}\right\rangle= & -\left\langle G_{\mathrm{BS}}\right\rangle_{\mathrm{i}}\left\{\frac{P_{\mathrm{i}}^{\prime}}{e n_{\mathrm{e}}}+\phi^{\prime}+L_{34}^{(0)} \frac{T_{\mathrm{i}}^{\prime}}{e Z_{\mathrm{eff}}}\right\} \\
+ & \left\{\frac{Z_{\mathrm{f}}}{Z_{\mathrm{eff}}}-Z_{\mathrm{f}}\left[1+\left(1+\frac{m_{\mathrm{i}}}{m_{\mathrm{f}}}\right)\left(\frac{v_{\mathrm{c}}}{\bar{u}_{\mathrm{f}}}\right)^{3}\right] N_{33}^{(1)}\right\} \frac{Z_{\mathrm{f}} n_{\mathrm{f}}}{n_{\mathrm{e}}}\left\langle B u_{/ / \mathrm{f}}\right\rangle \\
- & \Lambda_{\mathrm{NC}}^{(0)} \frac{e \tau_{\mathrm{ei}}}{m_{\mathrm{e}}}\left\langle B E_{/ /}^{(A)}\right\rangle \\
\left\langle B u_{/ / \mathrm{i}}\right\rangle= & -\left\langle G_{\mathrm{BS}}\right\rangle_{\mathrm{i}}\left\{\frac{P_{\mathrm{i}}^{\prime}}{e n_{\mathrm{e}}}+\phi^{\prime}+L_{34}^{(0)} \frac{T_{\mathrm{i}}^{\prime}}{e Z_{\mathrm{eff}}}\right\} \\
& -Z_{\mathrm{f}}\left[1+\left(1+\frac{m_{\mathrm{i}}}{m_{\mathrm{f}}}\right)\left(\frac{v_{\mathrm{c}}}{\bar{u}_{\mathrm{f}}}\right)^{3}\right] N_{33}^{(1)} \frac{Z_{\mathrm{f}} n_{\mathrm{f}}}{n_{\mathrm{e}}}\left\langle B u_{/ / \mathrm{f}}\right\rangle \\
& +Z_{\mathrm{eff}} L_{11}^{(0)} N_{33}^{(1)} \frac{e \tau_{\mathrm{ei}}}{m_{\mathrm{e}}}\left\langle B E_{/ /}^{(A)}\right\rangle, \\
\left\langle B J_{/ /}\right\rangle= & \left\{1-\frac{Z_{\mathrm{f}}}{Z_{\mathrm{eff}}}\right\}\left\langle B J_{/ / \mathrm{f}}\right\rangle+\Lambda_{\mathrm{NC}}^{(0)} \frac{e^{2} n_{\mathrm{e}} \tau_{\mathrm{ei}}}{m_{\mathrm{e}}}\left\langle B E_{/ /}^{(A)}\right\rangle,
\end{aligned}
$$

where

$$
\begin{aligned}
L_{11}^{(0)} & =\left\{\bar{\mu}_{\mathrm{e} 1} \bar{l}_{22}^{\mathrm{ee}}-\bar{\mu}_{\mathrm{e} 2} \bar{l}_{12}^{\mathrm{ee}}\right\} \frac{1}{D_{\mathrm{e} 1}}, \\
L_{34}^{(0)} & =-\frac{\bar{\mu}_{\mathrm{i} 2}}{\bar{\mu}_{\mathrm{i} 1}}, \\
N_{33}^{(1)} & =-\frac{\varepsilon}{\bar{\mu}_{\mathrm{i} 1}}, \\
\Lambda_{\mathrm{NC}}^{(0)} & =\bar{l}_{11}^{\mathrm{ee}} \bar{l}_{22}^{\mathrm{ee}} \frac{1}{D_{\mathrm{e} 1}}, \\
D_{\mathrm{e} 1} & =\bar{l}_{11}^{\mathrm{ee}} \bar{l}_{22}^{\mathrm{ee}}-\left(\bar{l}_{12}^{\mathrm{ee}}\right)^{2} .
\end{aligned}
$$

In Eq. (20), $L_{11}^{(0)}$ and $N_{33}^{(1)}$ become the order of $\varepsilon$ and the order of unity, respectively, because $\bar{\mu}_{\mathrm{e} k} \sim \bar{\mu}_{\mathrm{i} k} \sim \varepsilon$. Equation (19) shows that the parallel neoclassical current is reduced to the usual classical parallel current, i.e., the classical beam-driven current with electron return current effect $-Z_{\mathrm{f}} / Z_{\text {eff }}$ and the classical Spitzer current. However, as is clear from Eqs. (17) and (18), the parallel flows of both electrons and ions are driven by the thermodynamical forces, fast ions, and the external electric field. Since the parallel flows driven by the thermodynamical forces are the same for electrons and ions in the leading order, the parallel current due to the thermodynamical force vanishes. The ion flow due to fast ions are canceled by that of electrons in Eq. (19). Thus, the momentum transferred from fast ions to thermal ions is larger than that to electrons by the order of $\varepsilon^{-2}$. Hence, although the expression of the parallel current is reduced to the classical one, neoclassical effects can be seen in the plasma rotation. The toroidal and poloidal rotations of ions are expressed using Eqs. (10), (11) and (18) as follows:

$$
\begin{aligned}
\left\langle\vec{u}_{\mathrm{i}} \cdot \nabla \zeta\right\rangle= & -\frac{J\left\langle G_{\mathrm{BS}}\right\rangle_{\mathrm{iN}}+t I}{t(J+t I)}\left(\frac{1}{e n_{\mathrm{e}}} \frac{\mathrm{d} P_{\mathrm{i}}}{\mathrm{d} \phi}+\frac{\mathrm{d} \phi}{\mathrm{d} \phi}\right)-\frac{J\left\langle G_{\mathrm{BS}}\right\rangle_{\mathrm{iN}} L_{34}^{(0)}}{t(J+t I)} \frac{1}{e Z_{\mathrm{eff}}} \frac{\mathrm{d} T_{\mathrm{i}}}{\mathrm{d} \phi} \\
& -\frac{Z_{\mathrm{f}}}{J+\iota I}\left[1+\left(1+\frac{m_{\mathrm{i}}}{m_{\mathrm{f}}}\right)\left(\frac{v_{\mathrm{c}}}{\bar{u}_{\mathrm{f}}}\right)^{3}\right] N_{33}^{(1)} \frac{Z_{\mathrm{f}} n_{\mathrm{f}}}{n_{\mathrm{e}}}\left\langle B u_{/ / \mathrm{f}}\right\rangle, \\
\left\langle\vec{u}_{\mathrm{i}} \cdot \nabla \theta\right\rangle= & \frac{J}{J+t I}\left[1-\left\langle G_{\mathrm{BS}}\right\rangle_{\mathrm{iN}}\right]\left(\frac{1}{e n_{\mathrm{e}}} \frac{\mathrm{d} P_{\mathrm{i}}}{\mathrm{d} \phi}+\frac{\mathrm{d} \phi}{\mathrm{d} \phi}\right)-\frac{J\left\langle G_{\mathrm{BS}}\right\rangle_{\mathrm{iN}} L_{34}^{(0)}}{J+t I} \frac{1}{e Z_{\mathrm{eff}}} \frac{\mathrm{d} T_{\mathrm{i}}}{\mathrm{d} \phi} \\
- & \frac{t Z_{\mathrm{f}}}{J+t I}\left[1+\left(1+\frac{m_{\mathrm{i}}}{m_{\mathrm{f}}}\right)\left(\frac{v_{\mathrm{c}}}{\bar{u}_{\mathrm{f}}}\right)^{3}\right] N_{33}^{(1)} \frac{Z_{\mathrm{f}} n_{\mathrm{f}}}{n_{\mathrm{e}}}\left\langle B u_{/ / \mathrm{f}}\right\rangle,
\end{aligned}
$$

where $\left\langle G_{\mathrm{BS}}\right\rangle_{\mathrm{iN}} \equiv\left\langle G_{\mathrm{BS}}\right\rangle_{\mathrm{i}} /\left\langle G_{\mathrm{BS}}\right\rangle_{\mathrm{T}} \leq 1\left(\left\langle G_{\mathrm{BS}}\right\rangle_{\mathrm{T}}\right.$ is the geometric factor for the axisymmetric toroidal systems $\left.{ }^{4}\right)$ and we have neglected the term due to the external inductive electric field, because $L_{11}^{(0)}$ is the order of $\varepsilon$. Because $\nabla \zeta \sim \boldsymbol{e}_{\zeta} / R$ and $\nabla \theta \sim \boldsymbol{e}_{\theta} / r$ where $\boldsymbol{e}_{\zeta}$ and $\boldsymbol{e}_{\theta}$ are the unit vectors in the toroidal and poloidal directions, respectively, and $R$ and $r$ are the major and 
minor radii, respectively, $R\left\langle\overrightarrow{\boldsymbol{u}}_{\mathrm{i}} \cdot \nabla \zeta\right\rangle$ and $r\left\langle\vec{u}_{\mathrm{i}} \cdot \nabla \theta\right\rangle$ have the physical meanings as the toroidal and poloidal rotations, respectively. The ion parallel flow due to fast ions in the PfirshSchlüter regime (Eq. (18)) is larger than that in the $1 / \nu$ regime (Eq. (14)) by the order of $\varepsilon^{-1}$. Thus, the ion toroidal and poloidal rotations due to fast ions become large as the ion collision frequency increases. Let us consider the two limiting cases in the non-axisymmetric toroidal system. In the non-axisymmetric toroidal system, the helical ripples due to non-axisymmetry make the normalized geometric factor $\left\langle G_{\mathrm{BS}}\right\rangle_{a \mathrm{~N}}$ significantly small ${ }^{4}$, and the total toroidal current $2 \pi I$ inside a flux surface is very small compared with the total poloidal current $2 \pi J$ outside a flux surface. In such the case, the toroidal rotation due to fast ions is predominant and Eq. (21) reduces to the following equation:

$$
\left\langle\vec{u}_{\mathrm{i}} \cdot \hat{e}_{\zeta}\right\rangle \simeq-\frac{R Z_{\mathrm{f}}}{J}\left[1+\left(1+\frac{m_{\mathrm{i}}}{m_{\mathrm{f}}}\right)\left(\frac{v_{\mathrm{c}}}{\bar{u}_{\mathrm{f}}}\right)^{3}\right] N_{33}^{(1)} \frac{Z_{\mathrm{f}} n_{\mathrm{f}}}{n_{\mathrm{e}}}\left\langle B u_{/ / \mathrm{f}}\right\rangle .
$$

In this case the thermodynamical contribution vanishes and the magnetic field dependence of the ion toroidal rotation can be seen only through the term of $N_{33}^{(1)}$ as follows:

$$
\left\langle\vec{u}_{\mathrm{i}} \cdot \hat{e}_{\zeta}\right\rangle \propto-N_{33}^{(1)} \propto \frac{1}{\bar{\mu}_{\mathrm{i} 1}},
$$

where $\bar{\mu}_{i 1}$ in the Pfirsh-Schlüter regime is given in Eq. (A. 12) in Appendix 3. Thus, the ion toroidal rotation is inversely proportional to the ion viscosity coefficient $\bar{\mu}_{i 1}$ in this limit. On the other hand, the poloidal rotation of ions reduces to

$$
\left\langle\vec{u}_{\mathrm{i}} \cdot \hat{e}_{\theta}\right\rangle \simeq r\left(\frac{1}{e n_{\mathrm{e}}} \frac{\mathrm{d} P_{\mathrm{i}}}{\mathrm{d} \phi}+\frac{\mathrm{d} \phi}{\mathrm{d} \phi}\right)-\frac{\operatorname{tr} Z_{\mathrm{f}}}{J}\left[1+\left(1+\frac{m_{\mathrm{i}}}{m_{\mathrm{f}}}\right)\left(\frac{v_{\mathrm{c}}}{\bar{u}_{\mathrm{f}}}\right)^{3}\right] N_{33}^{(1)} \frac{Z_{\mathrm{f}} n_{\mathrm{f}}}{n_{\mathrm{e}}}\left\langle B u_{/ / \mathrm{f}}\right\rangle .
$$

In this case, the thermodynamical contribution remains.

In $\mathrm{CHS}^{1)}$ central helical ripples along the magnetic axis can vary according to the outward or inward shift of the plasma. If $R_{\text {axis }}=90 \sim 95 \mathrm{~cm}$, where $R_{\text {axis }}$ is the major radius of the magnetic axis in the vacuum field, the central helical ripples are negligibly small and they increase sharply if $R_{\text {axis }}$ exceeds $95 \mathrm{~cm}$. In the vicinity of the region where the helical ripples are vanishingly small the system can be thought to be nearly axisymmetric and $\left\langle G_{\mathrm{BS}}\right\rangle_{\mathrm{iN}} \simeq 1$. In such the case Eqs. (21) and (22) become

$$
\begin{gathered}
\left\langle\vec{u}_{\mathrm{i}} \cdot \hat{e}_{\zeta}\right\rangle \simeq-\frac{R}{t}\left(\frac{1}{e n_{\mathrm{e}}} \frac{\mathrm{d} P_{\mathrm{i}}}{\mathrm{d} \phi}+\frac{\mathrm{d} \phi}{\mathrm{d} \phi}\right)-\frac{R L_{34}^{(0)}}{t} \frac{1}{e Z_{\text {eff }}} \frac{\mathrm{d} T_{\mathrm{i}}}{\mathrm{d} \phi} \\
-\frac{R Z_{\mathrm{f}}}{J}\left[1+\left(1+\frac{m_{i}}{m_{\mathrm{f}}}\right)\left(\frac{v_{\mathrm{c}}}{\bar{u}_{\mathrm{f}}}\right)^{3}\right] N_{33}^{(1)} \frac{Z_{\mathrm{f}} n_{\mathrm{f}}}{n_{\mathrm{e}}}\left\langle B u_{/ / \mathrm{f}}\right\rangle, \\
\left\langle\vec{u}_{\mathrm{i}} \cdot \hat{e}_{\theta}\right\rangle \simeq-r L_{34}^{(0)} \frac{1}{e Z_{\text {eff }}} \frac{\mathrm{d} T_{\mathrm{i}}}{\mathrm{d} \phi}-\frac{t r Z_{\mathrm{f}}}{J}\left[1+\left(1+\frac{m_{\mathrm{i}}}{m_{\mathrm{f}}}\right)\left(\frac{v_{\mathrm{c}}}{\bar{u}_{\mathrm{f}}}\right)^{3}\right] N_{33}^{(1)} \frac{Z_{\mathrm{f}} n_{\mathrm{f}}}{n_{\mathrm{e}}}\left\langle B u_{/ / \mathrm{f}}\right\rangle .
\end{gathered}
$$

As the gradients of the ion pressure and temperature, and electrostatic potential with respect to $\phi$, i.e., $\mathrm{d} P_{\mathrm{i}} / \mathrm{d} \phi, \mathrm{d} T_{\mathrm{i}} / \mathrm{d} \phi$, and $\mathrm{d} \phi / \mathrm{d} \phi$ do not vanish on the magnetic axis, the thermodynamical contribution to toroidal and poloidal rotations of ions should not be neglected in the non-axisymmetric toroidal system with axisymmetry near the magnetic axis. Note that what is mentioned on the toroidal rotation is applicable to the parallel flow.

Finally, we consider the situation where electrons exist in the $1 / \nu$ collisionality regime and ions exist in the Pfirsh-Schlüter regime. This situation is realized by setting $\bar{\mu}_{\mathrm{e} k} \gg \bar{\mu}_{\mathrm{i} k} \sim \varepsilon$. In this case, the expressions of Eqs. (7)-(12) do not change, except for

$$
L_{34}^{(0)}=-\frac{\bar{\mu}_{\mathrm{i} 2}}{\bar{\mu}_{\mathrm{i} 1}}, \quad N_{33}^{(1)}=-\frac{\varepsilon}{\bar{\mu}_{\mathrm{i} 1}} .
$$

Note that the coefficients including $N_{33}^{(1)}$ must be evaluated using $N_{33}^{(1)}$ in Eq. (28). In the case where ions exist in the high collisionality regime $\left(\bar{\mu}_{\mathrm{i} k} \sim \varepsilon\right)$, the terms with superscript (1) become the same order as the terms with superscipt $(0)$. Thus, the ion parallel flow due to fast ions and the external inductive field becomes the same order as that of electrons. As a result 
of it, the momentum gained by ions is larger than that of electrons by the order of $\varepsilon^{-2}$. This result still holds when the direct interaction between fast ions and thermal ions is negligible, i.e., $\left(v_{\mathrm{c}} / \bar{u}_{\mathrm{f}}\right)^{3} \ll 1$.

\section{Conclusion and Discussion}

The effects of the external sources, i.e., fast ions due to the neutral beam injection and the external inductive electric field, on the neoclassical flow, current, and ion toroidal rotation are examined intensively. When both electrons and ions exist in the $1 / \nu$ or banana collisionality regime, light electrons carry the current attributed to fast ions and the external inductive field, while the heavy ions carry the momentum transferred from fast ions. It should be noted that the parallel ion flow due to the fast ions is smaller than the parallel electron flow due to fast ions by the order of $\sqrt{m_{\mathrm{e}} / m_{\mathrm{i}}}$. As the ion collision frequency increases, however, the situation becomes complicated, the reason of which comes from the momentum conservation of the Coulomb collision and the property of viscosity coefficients decreasing sharply with increasing collision frequency. Especially, when both electrons and ions exist in the PfirshSchlüter regime, the parallel neoclassical current reduces to the usual classical current. However, the parallel ion flow due to fast ions becomes the same order as that of electrons, and the momentum transferred from fast ions to thermal ions is larger than that to electrons by the order of $m_{\mathrm{i}} / m_{\mathrm{e}}$. As a result of it, the ion toroidal rotation is predominated by the parallel flow due to fast ions and the contribution of the thermodynamical forces is negligible in the nonaxisymmetric toroidal configuration with the significantly small normalized geometric factor or large helical ripples. Thus, the ion toroidal rotation is inversely proportional to the ion viscosity coefficient. If axisymmetry is recovered near the magnetic axis by shifting the plasma as is the case of CHS under an operation condition, the contribution of the thermodynamical forces remains there.

The results which we have obtained for the contribution of the external momentum sources to the parallel flow, current, and rotation in the Pfirsh-Schlüter collisionality regime may be qualitatively applicable even to the plateau regime if the quantities in the PfirshSchlüter regime are replaced by those in the plateau regime, because viscosity coefficients in the plateau regime are enough small compared with $1 / \nu$ or banana regime.

\section{Appendix 1. Expressions for the Flux-Surface-Averaged Parallel Quantities in Eq. (1)}

The flux-surface-averaged parallel friction $\left\langle\boldsymbol{B} \cdot \boldsymbol{F}_{a 1}\right\rangle$ and the heat friction $\left\langle\boldsymbol{B} \cdot \boldsymbol{F}_{a 2}\right\rangle$ with thermal species are given by ${ }^{3)}$

$$
\left[\begin{array}{r}
\left\langle\boldsymbol{B} \cdot \boldsymbol{F}_{a 1}\right\rangle \\
-\left\langle\boldsymbol{B} \cdot \boldsymbol{F}_{a 2}\right\rangle
\end{array}\right]=\sum_{b=0}^{N}\left[\begin{array}{ll}
l_{11}^{a b} & l_{12}^{a b} \\
l_{21}^{a b} & l_{22}^{a b}
\end{array}\right]\left[\begin{array}{r}
\left\langle B u_{/ / b}\right\rangle \\
-\frac{5}{2} \frac{\left\langle B q_{/ / b}\right\rangle}{P_{b}}
\end{array}\right]
$$

where $l_{i j}^{a b}(i, j=1,2, a, b=0, \cdot, \cdot, N)$ are the friction coefficients between species $a$ and $b(a=0$ for electrons and $a \neq 0$ for ion species). Since the friction coefficients are independent of both the magnetic field $\boldsymbol{B}$ and the collisionality regime, the expressions in Eq. (A. 1) for parallel friction forces in terms of the parallel flow and heat flux are the same between axisymmetric and non-axisymmetric toroidal systems in all collisonality regimes. The friction coefficients $l_{i j}^{a b}(i, j=1,2, a, b=0 \sim N)$ have the following relations:

$$
\begin{gathered}
l_{i j}^{a b}=l_{j i}^{b a} \\
\sum_{b=0}^{N} l_{i 1}^{a b}=0 \quad(i=1,2),
\end{gathered}
$$

which ensure the total momentum conservation of the Coulomb collision operator, i.e., $\sum_{b=0}^{N} \boldsymbol{F}_{a 1}$ $=0$.

The flux-surface-averaged parallel friction and head friction with fast ions are given by ${ }^{3)}$ 


$$
\begin{aligned}
& {\left[\begin{array}{r}
\left\langle\boldsymbol{B} \cdot \boldsymbol{F}_{0 \mathrm{f} 1}\right\rangle \\
-\left\langle\boldsymbol{B} \cdot \boldsymbol{F}_{0 \mathrm{f} 2}\right\rangle
\end{array}\right]=\frac{n_{\mathrm{e}} m_{\mathrm{e}}}{\tau_{\mathrm{ee}}} \frac{Z_{\mathrm{f}}^{2} n_{\mathrm{f}}}{n_{\mathrm{e}}}\left[\begin{array}{l}
1 \\
3 \\
\overline{2}
\end{array}\right]\left\langle B u_{/ \mathrm{ff}}\right\rangle \quad \text { for electrons, }} \\
& \left\langle\boldsymbol{B} \cdot \boldsymbol{F}_{a \mathrm{f} 1}\right\rangle=\frac{v_{\mathrm{c}}^{3}}{\bar{u}_{\mathrm{f}}^{3}} \frac{n_{a} e_{a}^{2}}{\sum_{b=1}^{N} \frac{n_{b} e_{b}^{2}}{m_{b}}}\left(\frac{1}{m_{\mathrm{f}}}+\frac{1}{m_{a}}\right)\left\langle\boldsymbol{B} \cdot \boldsymbol{F}_{0 \mathrm{f} 1}\right\rangle \text { for ion species, }
\end{aligned}
$$

where

$$
v_{\mathrm{c}}^{3}=\frac{3 \sqrt{\pi}}{4} \frac{m_{\mathrm{e}}}{m_{\mathrm{f}}} \sum_{b=1}^{N} \frac{n_{b} e_{b}^{2} m_{f}}{n_{e} m_{b}} v_{\mathrm{T}_{\mathrm{e}}}^{3}, \quad \frac{1}{\bar{u}_{\mathrm{f}}^{3}} \equiv \frac{\int \frac{v_{\prime \prime}}{v^{3}} f_{\mathrm{f}} \mathrm{d}^{3} v}{\int v_{\| \prime} f_{\mathrm{f}} \mathrm{d}^{3} v} .
$$

The quantities with subscript $f$ denote those which belong to fast ions, $v_{c}$ is the critical velocity at which the drag exerted by the electrons on the fast ion beam is equal to that of background ions on the fast ion beam. Here the conditions $v_{\mathrm{T}_{\mathrm{e}}} \gg\left|\vec{u}_{\mathrm{f}}\right| \gg v_{\mathrm{T}_{a}}$ for $a=1 \sim N$ have been assumed where $\vec{u}_{\mathrm{f}}$ is the beam velocity, $v_{\mathrm{T}_{\mathrm{e}}}$ the thermal velocity of electrons and $v_{\mathrm{T}_{a}}$ the thermal velocity of $a$ species ions. The heat friction of thermal ions with fast ions has been neglected.

The flux-surface-averaged parallel viscosity and the parallel heat viscosity have the following general form

$$
\left[\begin{array}{c}
\left\langle\boldsymbol{B} \cdot \nabla \cdot \Pi_{a}\right\rangle \\
-\left\langle\boldsymbol{B} \cdot \nabla \cdot \Theta_{a}\right\rangle
\end{array}\right]=\left[\begin{array}{ll}
\mu_{a 1} & \mu_{a 2} \\
\mu_{a 2} & \mu_{a 3}
\end{array}\right]\left[\begin{array}{c}
\left\langle B u_{/ / a}\right\rangle+\left\langle G_{\mathrm{BS}}\right\rangle_{a}\left(\frac{P_{a}^{\prime}}{e_{a} n_{a}}+\phi^{\prime}\right) \\
-\frac{2}{5} \frac{\left\langle B q_{/ / a}\right\rangle}{P_{a}}-\left\langle G_{\mathrm{BS}}\right\rangle_{a} \frac{T_{a}^{\prime}}{e_{a}}
\end{array}\right],
$$

where $\mu_{a j}(j=1 \sim 3)$ are viscosity coefficients and $\left\langle G_{\mathrm{BS}}\right\rangle_{a}$ is the geometric factor, and the prime denotes $\mathrm{d} / \mathrm{d} V$ with $V$ being the volume. The viscosity coefficients $\mu_{a j}$ have the geometrical dependences of the magnetic field and depend on the collisionality regime. The magnitude of them sharply deceases as the collision frequency inceases without regard to the existence of axisymmetry. However, the gepmetric factor $\left\langle G_{\mathrm{BS}}\right\rangle_{a}$ is deeply affected by whether the axisymmetry exists or not. In the axisymmetric toroidal system it has the common expression to all particle species in all collisionality regimes, which is expressed $\left\langle G_{\mathrm{BS}}\right\rangle_{\mathrm{T}}{ }^{4}$. In the non-axisymmetric toroidal systems, however, it depends on the collisionality regime where the particle species $a$ exists and is smaller than the axisymmetric one, i.e., $\left\langle G_{\mathrm{BS}}\right\rangle_{a} \leq\left\langle G_{\mathrm{BS}}\right\rangle_{\mathrm{T}}$. The magnitude of it roughly decreases as the collision frequency increases. The asymptotic forms for $\left\langle G_{\mathrm{BS}}\right\rangle_{a}$ in each collisionality regime can be seen in ref. 4 .

\section{Appendix 2. Definitions of Quantities in Eq. (2)}

The quantities in Eq. (2) are defied as follows for $(a, b=0 \sim N)$ :

$$
\begin{aligned}
& \boldsymbol{F}_{t}: F_{t 2 a+1} \equiv\left\langle\boldsymbol{B} \cdot \boldsymbol{F}_{a 1}\right\rangle, \\
& \boldsymbol{F}_{f}: F_{f 2 a+1} \equiv\left\langle\boldsymbol{B} \cdot \boldsymbol{F}_{a \mathrm{f} 1}\right\rangle, \\
& \boldsymbol{E}_{\mathrm{A}}: E_{\mathrm{A} 2 a+1} \equiv e_{a} n_{a}\left\langle\boldsymbol{B} \cdot \boldsymbol{E}^{(A)}\right\rangle, \\
& F_{t 2 a+2} \equiv-\left\langle\boldsymbol{B} \cdot \boldsymbol{F}_{a 2}\right\rangle, \\
& F_{f 2 a+2} \equiv-\left\langle\boldsymbol{B} \cdot \boldsymbol{F}_{a \mathrm{f} 2}\right\rangle \delta_{a 0}, \\
& \boldsymbol{V}: V_{2 a+1} \equiv\left\langle\boldsymbol{B} \cdot \boldsymbol{\nabla} \cdot \Pi_{a}\right\rangle, \\
& E_{A 2 a+2} \equiv 0 \text {, } \\
& G: G_{2 a+1} \equiv\left\langle G_{\mathrm{BS}}\right\rangle_{a}\left(\frac{P_{a}^{\prime}}{e_{a} n_{a}}+\phi^{\prime}\right), \quad G_{2 a+2} \equiv-\left\langle G_{\mathrm{BS}}\right\rangle_{a} \frac{T_{a}^{\prime}}{e_{a}}, \\
& X: X_{2 a+1} \equiv\left\langle B u_{/ / a}\right\rangle \text {, } \\
& X_{2 a+2} \equiv-\frac{2}{5} \frac{\left\langle B q_{1 / a}\right\rangle}{P_{a}}, \\
& L^{a b} \equiv\left[\begin{array}{ll}
l_{11}^{a b} & l_{12}^{a b} \\
l_{21}^{a b} & l_{22}^{a b}
\end{array}\right], \quad L_{t} \equiv\left[\begin{array}{cccc}
L^{00} & L^{01} & \cdot & L^{0 N} \\
L^{10} & L^{11} & \cdot & L^{1 N} \\
\cdot & \cdot & \cdot & \cdot \\
L^{N 0} & L^{N 1} & \cdot & L^{N N}
\end{array}\right],
\end{aligned}
$$




$$
\mu_{a} \equiv\left[\begin{array}{ll}
\mu_{a 1} & \mu_{a 2} \\
\mu_{a 2} & \mu_{a 3}
\end{array}\right], \quad \mu \equiv\left[\begin{array}{llll}
\mu_{0} & & & \\
& \mu_{1} & \\
& & & \\
& & & \mu_{N}
\end{array}\right],
$$

$\delta_{a 0}=1$ for $a=0,=0$ for $a \neq 0$.

\section{Appendix 3. Friction and Viscosity Coefficients}

In this Appendix we give friction and viscosity coefficients for a simple plasma consisting of electrons and one species ions. The normalized friction coefficients up to the order of $\varepsilon$ are given by

$$
\begin{array}{ll}
\bar{l}_{11}^{e e}=-Z_{\text {eff }}, & \bar{l}_{12}^{e e}=-\frac{3}{2} Z_{\text {eff }}, \\
\bar{l}_{22}^{e e}=-\left(\sqrt{2}+\frac{13}{4} Z_{\text {eff }}\right), & \bar{l}_{22}^{i i}=-\left(\sqrt{2}+\frac{15}{2 Z_{\text {eff }}} \frac{v_{\mathrm{T}_{\mathrm{i}}}}{v_{\mathrm{T}_{\mathrm{e}}}}\right),
\end{array}
$$

where $v_{\mathrm{T}_{\mathrm{e}}}$ and $v_{\mathrm{T}_{\mathrm{i}}}$ are thermal velocities of electrons and ions, respectively, therefore, $v_{\mathrm{T}_{\mathrm{i}}} / v_{\mathrm{T}_{\mathrm{e}}}$ $\sim Z_{\text {eff }}^{2} \varepsilon$.

The viscosity coefficients up to the order of $\varepsilon$ are:

for the $1 / \nu$ or banana collisionality regime

$$
\begin{aligned}
& \bar{\mu}_{a 1}=\frac{f_{\mathrm{t}}}{f_{\mathrm{c}}}\left\{\sqrt{2}-\ln (1+\sqrt{2})+c_{a}\right\}, \\
& \bar{\mu}_{a 2}=\frac{f_{\mathrm{t}}}{f_{\mathrm{c}}}\left\{2 \sqrt{2}-\frac{5}{2} \ln (1+\sqrt{2})+\frac{3}{2} c_{a}\right\}, \\
& \bar{\mu}_{a 3}=\frac{f_{\mathrm{t}}}{f_{\mathrm{c}}}\left\{\frac{39}{8} \sqrt{2}-\frac{25}{4} \ln (1+\sqrt{2})+\frac{13}{4} c_{a}\right\},
\end{aligned}
$$

for the plateau collisionality regime

$$
\bar{\mu}_{a 1}=\frac{\lambda_{a}}{\lambda_{P L}} 2, \quad \bar{\mu}_{a 2}=-\frac{\lambda_{a}}{\lambda_{P L}}, \quad \bar{\mu}_{a 3}=\frac{\lambda_{a}}{\lambda_{P L}} \frac{13}{2},
$$

for the Pfirsh-Schlüter collisionality regime

$$
\begin{aligned}
& \bar{\mu}_{a 1}=\left(\frac{\lambda_{a}}{\lambda_{P S}}\right)^{2} \frac{205 \sqrt{2} / 32+c_{a} 51 / 4}{267 / 40+c_{a} 903 / 80+c_{a}^{2} 36 / 5}, \\
& \bar{\mu}_{a 2}=-\left(\frac{\lambda_{a}}{\lambda_{P S}}\right)^{2} \frac{331 \sqrt{2} / 32+c_{a} 57 / 2}{267 / 40+c_{a} 903 / 80+c_{a}^{2} 36 / 5}, \\
& \bar{\mu}_{a 3}=\left(\frac{\lambda_{a}}{\lambda_{P S}}\right)^{2} \frac{1045 \sqrt{2} / 32+c_{a} 81}{267 / 40+c_{a} 903 / 80+c_{a}^{2} 36 / 5},
\end{aligned}
$$

where $c_{a}=Z_{\text {eff }}$ for elecsrons $(a=\mathrm{e})$ and 0 for ions $(a=i)$. The fraction of trapped particles $f_{\mathrm{t}}$ and untrapped particles $f_{\mathrm{c}}$, the mean free path $\lambda_{a}$ of the particle species $a$, and the characteristic length of the magnetic field in the plateau regime $\lambda_{\mathrm{PL}}$ and in the Pfirsh-Schlüter regime $\lambda_{\mathrm{PS}}$ are given by

$$
\begin{gathered}
f_{\mathrm{t}}=1-\frac{3}{4} \frac{\left\langle B^{2}\right\rangle}{B_{\max }^{2}} \int_{0}^{1} \frac{1}{\left[\sqrt{1-\lambda \frac{B}{B_{\max }}}\right]} \lambda \mathrm{d} \lambda, \\
f_{\mathrm{c}}=1-f_{\mathrm{t}}, \quad \lambda_{a}=\tau_{a a} v_{T a}, \\
\frac{1}{\lambda_{\mathrm{PL}}} \equiv \frac{\Phi_{\mathrm{T}}^{\prime}\left(t \mu_{\mathrm{p}}+\mu_{\mathrm{t}}\right)}{\left\langle B^{2}\right\rangle}, \quad \frac{1}{\lambda_{\mathrm{PS}}{ }^{2}} \equiv \frac{3}{2} \frac{\left\langle(\boldsymbol{n} \cdot \nabla B)^{2}\right\rangle}{\left\langle B^{2}\right\rangle},
\end{gathered}
$$

where $\tau_{a a}$ is the Braginskii collision time: $\tau_{a a}^{-1}=4 /(3 \sqrt{\pi}) \cdot 4 \pi n_{a} e_{a}^{4} \ln \Lambda /\left(m_{a}^{2} v_{T a}^{3}\right)$, 


$$
t \mu_{\mathrm{p}}+\mu_{\mathrm{t}}=-\frac{\sqrt{\pi}}{2}\left[\frac{\boldsymbol{n} \cdot \nabla B}{\Phi_{\mathrm{T}}} \sum_{m \neq 0, n \neq 0} \frac{(t m+n)}{|t m+n|} B_{m n}^{\mathrm{H}} \sin \left[2 \pi\left(m \theta_{\mathrm{H}}+n \zeta_{\mathrm{H}}\right)\right]\right] .
$$

$B_{m n}^{\mathrm{H}}$ is the spectrum of $B$ in the Hamada coordinates, i.e., $B=\sum_{m n} B_{m n}^{\mathrm{H}} \cos \left[2 \pi\left(m \theta_{\mathrm{H}}+n \zeta_{\mathrm{H}}\right)\right]$. The spectrum of $B$ in the Hamada coordinates is easily obtained from the Boozer coordinates as shown in ref. 4.

\section{REFERENCES}

1) K. Nishimura, K. Matsuoka, M. Fujiwara, K. Yamazaki, J. Todoroki, T. Kaminura, T. Amano, H. Sanuki, S. Okamura, M. Hosokawa, H. Mamada, S. Tanahashi, S. Kubo, Y. Takita, T. Shoji, O. Kaneko, H. Iguchi and C. Takahashi: Fusion Tech. 17 (1990) 86.

2) K. Ida, H. Yamada, H. Iguchi, K. Itoh and CHS Group: Phys. Rev. Letters 67 (1991) 58.

3) S.P. Hirshman and D.J. Sigmar: Nucl. Fusion 21 (1981) 1079.

4) N. Nakajima and M. Okamoto: J. Phys. Soc. Jpn. 61 (1992) 833.

5) K.C. Shaing and J.D. Callen: Phys. Fluid 26 (1983) 3315.

6) K.C. Shaing, S.P. Hirshman and J.D. Callen: Phys. Fluid 29 (1986) 521.

7) M. Coronado and H. Wobig: Phys. Fluid 29 (1986) 527.

8) N. Nakajima and M. Okamoto: J. Phys. Soc. Jpn. 60 (1991) 4146. 